\title{
L'ecocardioscopia nella stratificazione cardiovascolare delle malattie renali croniche: il Nefrologo Protagonista
}

\author{
Rodolfo F. Rivera ${ }^{1}$, Antonio De Pascalis ${ }^{2}$, Fulvio Floccari ${ }^{3}$, Luca Di Lullo ${ }^{4}$ \\ ${ }^{1}$ U.O. Nefrologia e Dialisi, Ospedale San Gerardo, ASST, Monza \\ ${ }^{2}$ U.O. Nefrologia e Dialisi, Ospedale Vito Fazzi, Lecce \\ ${ }^{3}$ U.O. Nefrologia e Dialisi, Ospedale San Paolo, Civitavecchia (RM) \\ ${ }^{4}$ U.O. Nefrologia e Dialisi, Ospedale L. Parodi-Delfino, Colleferro (RM)
}

\begin{abstract}
Echocardioscopy in cardiovascular stratification of chronic kidney diseases: the nephrologist is the protagonist Cardiovascular disease (CV) is the leading cause of morbidity and mortality in patients with chronic kidney disease (CKD). The increase in cardiac complications in patients with CKD has encouraged nephrologists to acquire the cardiac diagnostic competences in the use of ultrasound. A formal echocardiographic approach requires standard times and the immediate availability of the cardiac-sonographer. Therefore, a goal-directed cardiac ultrasound and a focused echocardiogram or echocardioscopy strategy were created specifically tailored to the non-cardiologists as well as internists, interventists and intensivists. The concept of echocardioscopy has been incompletely evaluated and it is necessary to find a curricular program to grant proficiency.

The introduction of echocardioscopy in the nephrologist's tasks has the advantage of widening the spectrum of the diagnostic of cardiac complications in CKD patients, but also confers several technical limitations.

The aim of this revision is to analyze the use of the cardiac ultrasound equipment by the nephrologists and the requirements for the echocardioscopy strategy. In addition, it will specify the advantages and the disadvantages of cardiac-diagnostic management by nephrologists suggesting the possible clinical settings for the application of this method.
\end{abstract}

Keywords: Echocardiography, Echocardioscopy, Focused cardiac ultrasound, Goal-directed echocardiogram

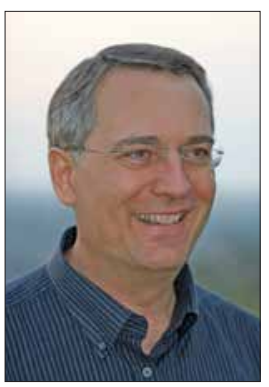

Rodolfo F. Rivera

\section{Introduzione}

La patologia cardiovascolare (CV) rappresenta la principale causa di morbidità e di mortalità nei pazienti affetti da malattie renali croniche (MRC) (1). L'Annual Data Report dell'USRDS (2) riporta che più del $38 \%$ dei decessi tra i pazienti in trattamento dialitico avviene come conseguenza di patologie CV. Dal punto di vista fisiopatologico, il fenomeno è la conseguenza dell'interazione tra

Accepted: May 12, 2016

Published online: May 26, 2016

Indirizzo per la corrispondenza:

Dr. Rodolfo F. Rivera

U.O. Nefrologia

Ospedale San Gerardo, ASST Monza

Via Pergolesi 33

20050 Monza

rodolfofrivera@gmail.com

fattori di rischio "tradizionali", come età, ipertensione arteriosa, diabete, dislipidemie, obesità, tabagismo e così via e quelli "specifici" dell'uremia, come anemia, iperomocisteinemia, disfunzione endoteliale e stato infiammatorio (3). Tuttavia, alcuni fattori tipici della MRC, come il controllo pressorio e la gestione della volemia, hanno un effetto diretto sul miocardio, generando alterazioni morfologiche e funzionali come I'ipertrofia ventricolare sinistra, il rimodellamento cardiaco, la dilatazione cavitaria, la fibrosi miocardica e lo scompenso sistolico-diastolico (4). Inoltre, i noti disturbi del metabolismo minerale e l'iperparatiroidismo secondario conducono alle calcificazione vascolari e valvolari (5), aumentando da 3 a 4 volte il rischio di mortalità $\mathrm{CV}$ degli uremici (6).

L'incremento di queste complicanze nella MRC ha progressivamente incoraggiato i nefrologi ad acquisire nel proprio background le competenze tecnico-strumentali per la diagnosi della patologia cardiaca. Infatti, sia sul territorio nazionale che all'estero, sono sempre più presenti strutture operative nefrologiche che si occupano di diagnostica cardiovascolare e di stratificazione di rischio di pazienti nefropatici. II ricorso all'ecocardiografia al di fuori degli ambienti cardiologici non è solo una prerogativa nefrologica, ma la tendenza è percepibile anche in altri settori come quelli internistici, generalistici e intensivisti. I 


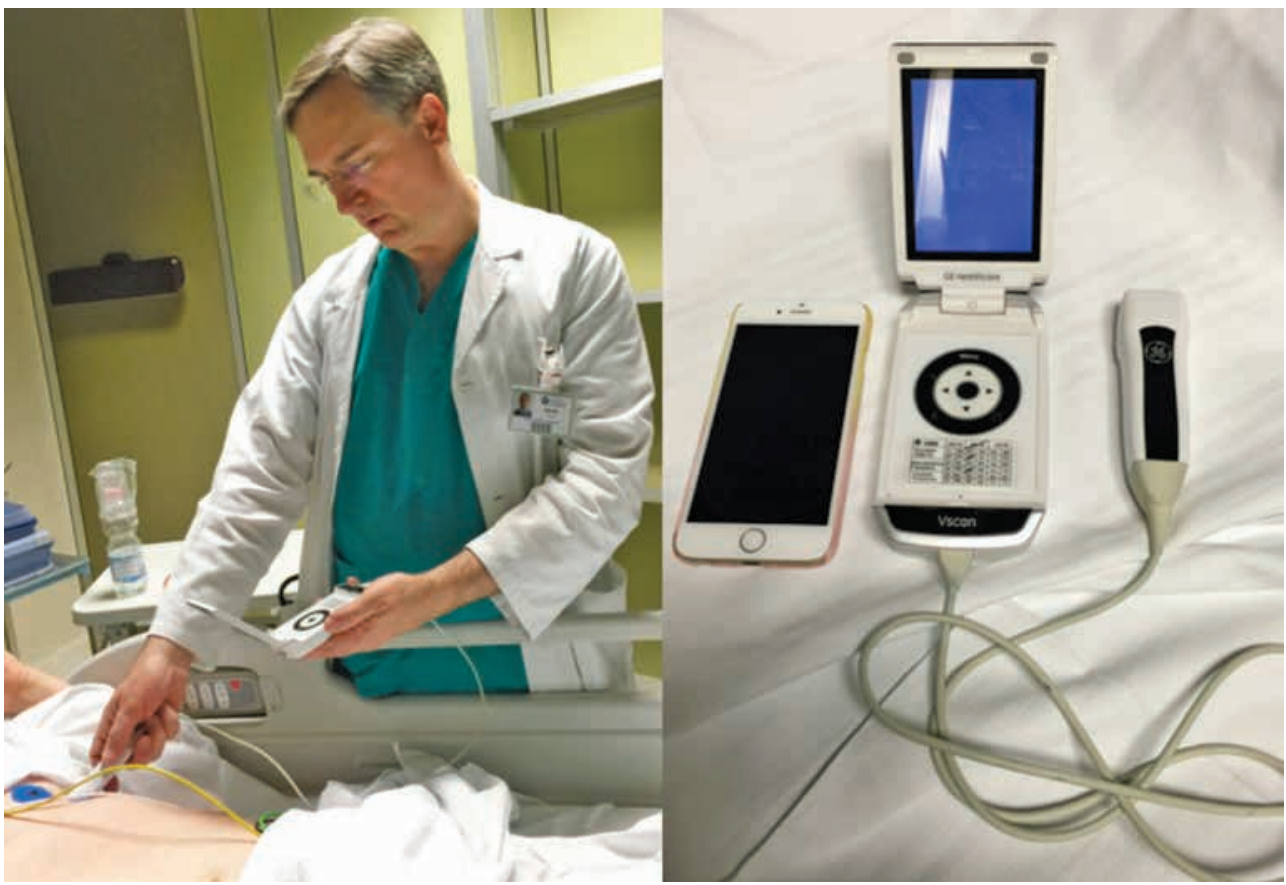

Fig. 1 - Apparecchiatura ecocardiografica portatile o da "tasca" direttamente al "bedside" del paziente.

principi dell'offerta e della domanda, applicati al settore sanitario, hanno ulteriormente innalzato questo interesse. L'offerta è data dall'evoluzione tecnologica e dallo sviluppo di nuove generazioni di apparecchiature a ultrasuoni con maggiore capacità di elaborazione e semplicità di utilizzo: oggi è sempre più fattibile disporre di strumenti più sofisticati e meno costosi, direttamente al "bedside" del paziente. La domanda, invece, è data dalle già note caratteristiche cliniche e anagrafiche dei nostri pazienti, con un sempre maggior numero di comorbidità e fattori di rischio di tipo $\mathrm{CV}$.

In questa revisione, verranno discussi gli utilizzi dell'apparecchiatura ecocardiografica da parte dei nefrologi o degli specialisti non cardiologi, requisiti necessari per l'esecuzione di un'ecocardioscopia, e l'analisi dei vantaggi e degli svantaggi della gestione diagnostica cardiovascolare da parte dei nefrologi e ci sarà un accenno ai possibili scenari per l'applicazione del metodo nel contesto specialistico.

\section{Ecocardiografia o ecocardioscopia?}

L'ecocardiografia color-Doppler transtoracica convenzionale (ETT) è diventata la principale metodica diagnostica in cardiologia, dato l'alto contenuto di informazioni, la disponibilità e la grande possibilità di espressione. La metodica, basata sugli ultrasuoni (US) nell'intervallo di frequenza fra 2 e massimo $10 \mathrm{MHz}$, presenta una varietà di vantaggi come la non invasività, la ripetibilità, la sicurezza biologica, la grande disponibilità con risultati immediati e i bassi costi operativi. Inoltre, i dispositivi portatili di ultima generazione, sempre più diffusi nei reparti internistici e in area critica (Fig. 1), hanno una dotazione tecnica molto affidabile, sono di facile utilizzo e consentono
TABELLA I - Requisiti indispensabili e opzionali per l'esecuzione di un esame ecocardiografico trastoracico

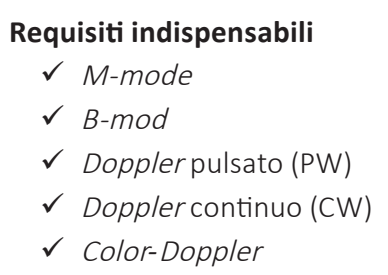

Requisiti opzionali
$\checkmark$ Elevato frame rate $(>150 \mathrm{~Hz})$
$\checkmark$ Anatomical M-mode
$\checkmark$ Dopplertissutale (TDI)
$\checkmark$ Automatic border detection
$\checkmark$ Caratterizzazione tissutale
$\checkmark$ 3-Dimensionale

l'esecuzione dell'esame direttamente al "bedside", limitando il disagio al paziente e gli inutili spostamenti di reparto. È opportuno osservare che, per la corretta esecuzione di uno studio ETT, sono necessari requisiti indispensabili (Tab. I), come l'impiego delle due modalità ( $M$-mode e $B$-mode) e l'utilizzo dell'eco-color-Doppler e dei sistemi Doppler continuo (CD) e pulsato (PD), utili per rilevare la direzione e la velocità del flusso ematico all'interno dei vasi. La Tabella I mostra anche i requisiti opzionali come il Doppler miocardico o tissutale (TDI), utili per rilevare la direzione e la velocità dei movimento delle pareti miocardiche. Inoltre, l'esame ETT comporta anche l'utilizzo di 


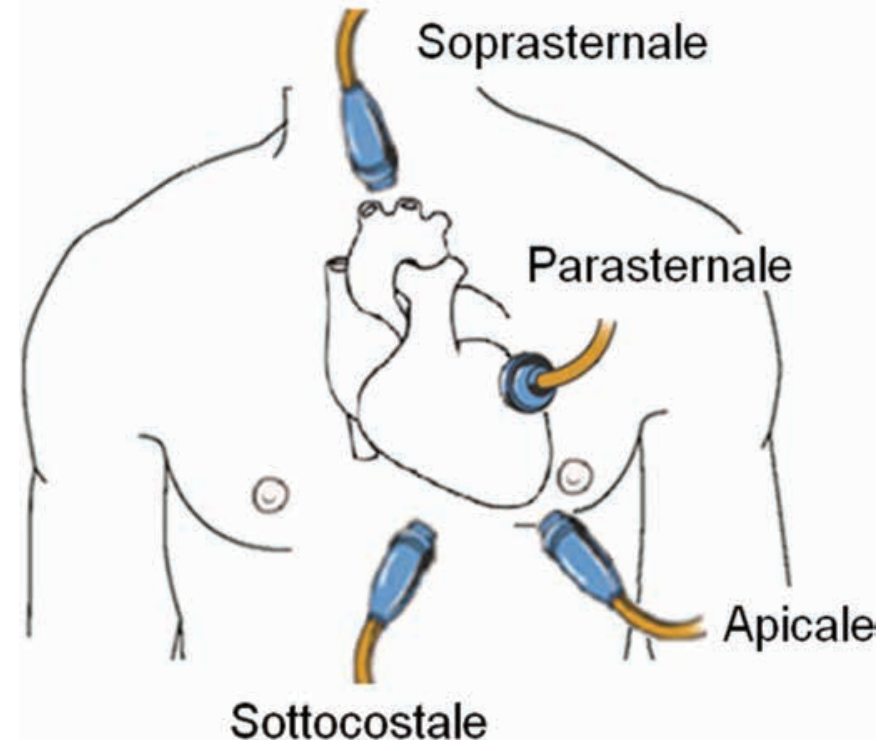

Fig. 2 - Proiezioni ecocardiografiche transtoraciche.

tutte le proiezioni ecocardiografiche transtoraciche illustrate nella Figura 2: parasternale asse lungo, parasternale asse corto, apicali (quattro, cinque, due e tre camere), sottocostale (asse corto e asse lungo) e soprasternale. L'uso sistematico di tutte le procedure descritte consente di diagnosticare la maggioranza delle patologia cardiache.

In generale, l'attuazione di un'ETT completa richiede tempi standard (mediamente 45 minuti, che possono aumentare nei casi più complessi) e la tempestiva reperibilità dell'ecocardiografista non sempre è garantita (7). Questo problema è particolarmente sentito negli ambienti intensivisti, dove il divario tra l'eccessiva domanda e la limitata offerta conosce la massima espressione.

Negli ultimi anni, la valutazione ecocardiografica del paziente emodinamicamente instabile da parte dell'intensivista non cardiologo si è sviluppata con un approccio "focale", "limitato" o "goal-directed echocardiogram (GDE)" (8). Questa strategia, nota anche come "ecocardioscopia" si sta rapidamente evolvendo in altre realtà specialistiche non cardiologiche, rivelandosi molto utile non solo per la diagnosi, ma anche per il monitoraggio e per la gestione clinica delle complicanze cardiache dei pazienti. Dato l'aumento delle richieste di ETT nella maggior parte delle discipline internistiche, associato alla continua esigenza dell'uso dell'ecocardiogramma da parte di specialisti non cardiologi, l'American Society of Echocardiography (ASE) ha definito, in un position paper, gli standard minimi di competenza per i tecnici ecocardiografici e per i medici non cardiologi che intendono interessarsi alla diagnostica ecografica cardiovascolare nel contesto delle loro specialità (9). L'ASE, inoltre, ha definito "Focus Cardiac Ultrasound" (FCU) l'esame eseguito dal medico specialista non cardiologo mirato alla valutazione del sistema cardiovascolare rappresentato da uno stretto elenco di potenziali diagnosi in ambienti clinici specifici (10). Anche l'European Association of Cardiovascular Imaging (EACVI) ha recentemente riconosciuto che i cardiologi non devono essere gli unici medici in grado di eseguire un FCU particolarmente in condizioni cliniche tipiche di pertinenza specialistica (11). In tal senso e a beneficio del paziente, l'EACVI incoraggia qualsiasi medico sufficientemente addestrato a ricavare una preziosa informazione diagnostica nel proprio contesto specialistico. Altre Società Scientifiche Nazionali hanno preso parte a questo dibattito internazionale. La Società Spagnola di Cardiologia (12), in un recente "position statement", stabilisce che l'ecocardioscopia è un metodo utile e agile per la valutazione morfologica e funzionale cardiaca, alla portata dei non cardiologi con un'adeguata formazione tecnica, che non richiede una regolare refertazione (potendo essere sufficiente un'annotazione sulla cartella del paziente) ed eseguibile con ecocardiografi portatili o da "tasca" (Fig. 1).

Nel contesto nazionale, da diversi anni ormai, la Società Italiana di Ecografia Cardiovascolare (SIEC) sta dedicando significative risorse ad approntare un programma formativo che ha come obiettivo la "Certificazione SIEC di competenza in ecocardiografia". Si tratta di un'esperienza originale, nata in Italia e cresciuta in parallelo a esperienze similari che si stanno evolvendo in altre Società Internazionali. Questo progetto, espresso inizialmente nella Consensus Conference del 2005 (13), ha permesso di legittimare e qualificare chi da anni si dedica quotidianamente all'applicazione clinica di questa metodica. Attualmente, l'offerta della SIEC riguarda la Competenza in ecocardiografia di base, la Competenza in ecocardiografia generale, la Competenza in alta specialità e il Corso di Eccellenza.

Nell'ultima revisione dell'Iter Formativo, la Società Italiana di Nefrologia (SIN) ha introdotto un nuovo capitolo nel corso avanzato di Ecografia per Nefrologi, intitolato "L'ecocardioscopia per il Nefrologo" (14). Questo particolare rappresenta un segnale di grande importanza e che conferma l'entità di attività di cui il nefrologo dovrà tenere conto nel prossimo futuro.

\section{Vantaggi e svantaggi dell'ecocardioscopia in nefrologia}

L'introduzione dell'ecografica cardiovascolare (anche se mirata) tra le mansioni dello specialista nefrologo, come proposto nel programma formativo della SIN, offre, da una parte, la possibilità di allargare in modo determinante lo spettro diagnostico delle complicanze dei nostri pazienti. Allo stesso tempo, però, l'introduzione di nuove tecniche e tecnologie espone gli specialisti a una serie di rischi e limitazioni dei quali bisogna tenere conto.

Come illustrato nella Tabella II esistono vantaggi e svantaggi nella gestione diagnostica cardiovascolare in ambienti non cardiologici. Tra i primi si riconoscono due tipi: i vantaggi clinici e quelli di carattere organizzativo.

I più rilevanti tra i vantaggi clinici sono il recupero dell'informazione in real-time della situazione morfologica e funzionale cardiaca e la possibilità di eseguire l'esame durante il ricovero 
TABELLA II - Vantaggi e svantaggi della gestione della diagnostica cardiovascolare in ambiente nefrologico

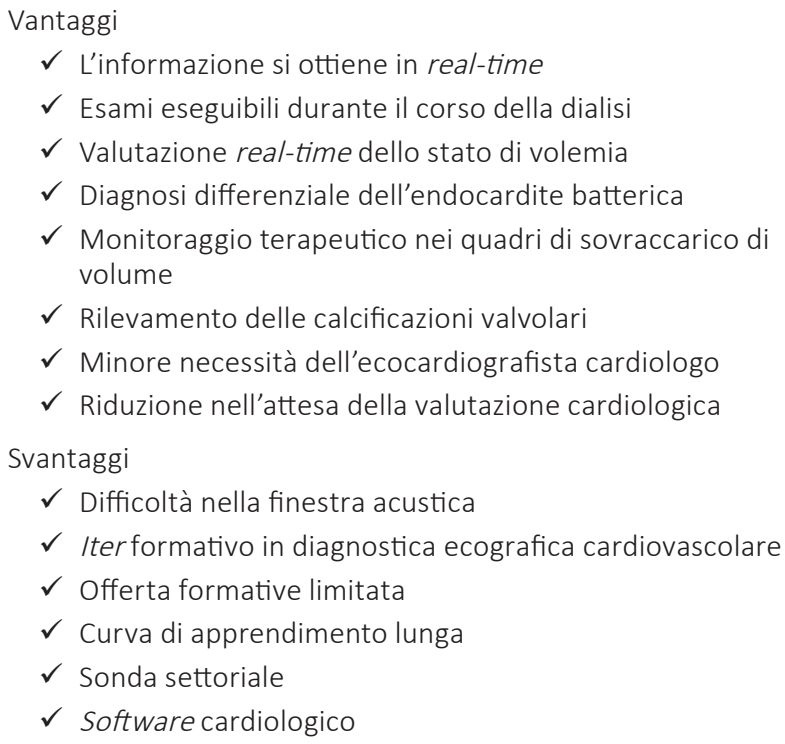

o, addirittura, il trattamento sostitutivo. La possibilità di verificare in maniera tempestiva lo stato della volemia e di monitorare nel tempo il risultato del trattamento impostato nei casi di sovraccarico acuto di volume rappresenta una valida opportunità di confronto multidisciplinare tra specialisti dalla quale il paziente non può che trarre beneficio. Inoltre, l'approccio ecocardiografico mirato e tempestivo delle valvole cardiache e dell'apparato sottovalvolare fornisce un contributo determinante per la diagnosi differenziale della sindrome febbrile di origine sconosciuta, come quelle osservate nell'endocardite batterica (in particolare nei dializzati portatori di cateteri venosi centrali transitori o a permanenza, pacemaker o defibrillatori). L'approccio valvolare consente anche il facile rilevamento delle aree di calcificazione e delle fibrocalcificazioni dei pazienti con iperparatiroidismo secondario, iperfosforemie o in terapia con chelanti calcici del fosforo.

Esistono almeno altri due vantaggi di tipo organizzativo. ॥ primo si riferisce alla minore "dipendenza" dell'ecocardiografista cardiologo, derivata dalla presenza di un addetto ecocardioscopista tra i nefrologi in grado di fornire le prime acquisizioni diagnostiche. II secondo si riferisce alla conseguente riduzione dell'attesa per la valutazione cardiologica, data proprio dalla precoce individuazione di un'ipotesi diagnostica, utile per il confronto interdisciplinare.

Queste realtà, però, sono più diffuse in ambienti intensivisti e un po' meno in quelli internistici (7-10), mentre appaiono ancora poco comuni nei contesti nefrologici. Esiste, effettivamente, una serie di svantaggi o limitazioni da considerare prima di investire in un'attività diagnostica di questa portata. Sarebbe auspicabile, per esempio, che la Divisione di Nefrologia che voglia iniziare un'attività di diagnostica cardiovascolare abbia già un background nell'utilizzo di tipo internistico e vascolare in ecografia. Nell'attualità, molti centri nefrologici hanno raggiunto un'assoluta autonomia diagnostica ecografica renale, dell'apparato urinario, internistica e vascolare, quest'ultima sia addominale (arterie renali e grossi vasi) che generale (tronchi sovra-aortici, arti superiori e arti inferiori). Queste sono le condizioni più opportune volte a evitare rischi derivati dall'inesperienza. Un percorso di diagnostica cardiovascolare, ritenuto, in nefrologia, di secondo livello (14), dovrebbe garantire, prima di tutto, le risorse necessarie per l'esecuzione di esami di tipo basico.

Tra gli svantaggi principali si segnalano quelli di tipo tecnico, come l'universale difficoltà nell'acquisizione di una finestra acustica adeguata. Questo punto, però, vale tanto per gli ecocardiografisti cardiologi che per quelli non cardiologi ed è l'esperienza dell'operatore che segna la differenza.

Un'importante limitazione riguarda l'aspetto formativo. Fermo restando l'elevato profilo qualitativo del corrente iter formativo per la diagnostica ecografica cardiovascolare a livello nazionale (13), l'aumento delle richieste da parte di specialisti non cardiologi potrebbe mettere alla prova questa offerta. In un contesto ideale, ogni nefrologo "ecografista" dovrebbe essere in grado di eseguire un'ecocardioscopia a seconda della situazione clinica del suo paziente. Sfortunatamente, però, non tutti i nefrologi "ecografisti" hanno ricevuto l'addestramento necessario e sono molto pochi quelli in possesso dell'accreditamento SIEC. Un ragionevole compromesso potrebbe, invece, vedere il nefrologo "ecografista" iniziare una valutazione ecografica del cuore nel tentativo di individuare il fenomeno fisiopatologico e/o eziologico alla base del problema, come si osserva nei casi di aumento della volemia, versamento pericardico o altri segni di scompenso congestizio (Fig. 3). Un'ecocardiografista con moderata esperienza o in corso di addestramento potrebbe facilmente rilevare alterazioni morfologiche e funzionali a carico del sistema valvolare (p. es., endocardite, calcificazioni valvolari: aorta, mitrale o tricuspide) oppure seguire nel tempo l'evoluzione del trattamento impostato per i quadri di scompenso congestizio. Contrariamente, nei casi clinici più complessi come alterazioni cinetiche segmentarie, disturbi nella funzione sistolica e diastolica del ventricolo sinistro e così via, sarebbe opportuno attendere l'intervento di un ecografista di maggiore esperienza che abbia completato l'addestramento ecocardiografico (13). La Figura 3 illustra l'associazione tra i diversi livelli di complessità della patologia cardiovascolare del paziente e la capacità operativa del nefrologo "ecografista", basata sul proprio livello di addestramento. L'addestramento ecocardiografico di uno specialista non cardiologo prevede una lunga curva di apprendimento e di verifica (13), che andrebbe considerata prima di fare una scelta.

Infine, è necessario ricordare la necessità di disporre di un'attrezzatura tecnica adeguata, come la sonda settoriale e un ecografo di recente generazione, in grado di supportare il software cardiologico. Nell'attualità, l'apparecchiatura standard 


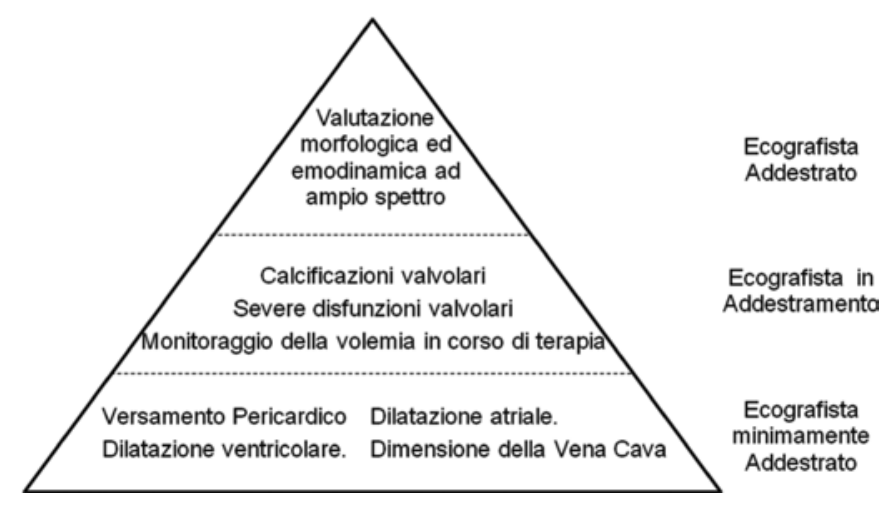

Fig. 3 - La piramide delle competenze dell'ecografista addestrato completamente, mediamente e minimamente in funzione delle possibilità e delle capacità diagnostiche.

prevede l'erogazione in uscita di materiale digitale facilitando l'archiviazione di grandi quantità di immagini. Questo approccio è molto vantaggioso per il basso costo, l'immediata applicabilità a qualunque ecocardiografo e il formato dei file ottenuti (.avi, .gif, ecc.), che sono manipolabili con software di utilizzo comune normalmente presenti sui computer più recenti.

L'ETT mirata e/o ecocardioscopia diventa, con il tempo, una tecnica relativamente facile da utilizzare, ma non è mai banale. Anche operatori esperti hanno dubbi, con una certa frequenza, ed e sempre bene chiedere una "second opinion", se possibile. II rischio di un approccio superficiale e di errate deduzioni cliniche non deve mai essere ignorato.

\section{Scenari di impiego dell'ecocardioscopia in nefrologia}

La nefrologia è una delle specialità internistiche più coinvolte nella gestione delle complicanze di carattere cardiovascolare. Dal punto di vista specialistico, esistono diversi scenari o situazioni cliniche nei quali il nefrologo ecografista può fornire il proprio contributo:

1. valutazione della funzione ventricolare: la valutazione accurata e tempestiva della funzione sistolica dovrebbe essere parte integrante della gestione medica di tutti $i$ pazienti nefropatici, in particolare di quelli che presentano un'instabilità emodinamica. Questo parametro viene spesso valutato qualitativamente mediante la sola ispezione visiva da parte dell'operatore esperto, ottenendo risultati molto affidabili (15). La sorveglianza della funzione sistolica nel tempo è consigliabile nei pazienti in trattamento dialitico cronico. Cambiamenti improvvisi della cinetica distrettuale del ventricolo sinistro, in grado di indurre un deficit funzionale ma non rilevabili clinicamente, suggeriscono una malattia coronarica in atto.

L'approccio inferiore o diaframmatico consente una rapida valutazione della funzione del ventricolo destro (VD). Dimensioni del VD superiori a $50 \mathrm{~mm}$ suggeriscono uno scompenso destro. Questa ipotesi viene confermata rilevando una riduzione della cinesi del VD ossia un valore inferiore ai $15 \mathrm{~mm}$ del TAPSE (tricuspid annular plane systolic excursion) (16);

2. valutazione delle dimensioni delle cavità cardiache: la valutazione in real-time delle dimensioni della cavità cardiaca può fornire un'importante informazione clinica utile per guidare le scelte terapeutiche. L'aumento delle dimensioni del ventricolo sinistro oltre i $55-60 \mathrm{~mm}$ suggerisce una miocardiopatia dilatativa. Se tale reperto è associato all'aumento delle dimensioni parietali (parete posteriore e setto interventricolare), il quadro più suggestivo è l'ipertrofia eccentrica del ventricolo sinistro. Queste caratteristiche morfologiche rendono necessaria l'analisi della funzione ventricolare sia in fase sistolica che in fase diastolica. Anche l'aumento delle dimensioni del ventricolo destro si associa a quadri di scompenso del piccolo circolo, da tenere in considerazione particolarmente nei pazienti dializzati con eccessi ponderali esagerati.

L'ecocardioscopia consente di stimare la morfologia e le dimensioni delle cavità atriali. La dilatazione dell'atrio sinistro (AS) è uno dei reperti più comuni rilevati nei pazienti dializzati. Gli incrementi ponderali intradialitici si associano all'aumento progressivo del volume atriale, che riveste una particolare importanza nei pazienti con fibrillazione atriale. L'età e l'ipertensione sono due noti fattori di rischio per la dilatazione dell'AS, ma esistono altre cause nei nefropatici, come l'insufficienza mitralica e la stenosi calcifica. La dilatazione dell'atrio destro, invece, è più associata alla patologia della valvola tricuspide o all'insorgenza di un'ipertensione polmonare;

3. valutazione dello stato di idratazione: il bilancio idrico è fondamentale nei pazienti dializzati, in quanto gli eccessi di volumi e l'esagerata rimozione di liquidi condizionano un'elevata mortalità. Uno dei sistemi più utili per la valutazione dello stato di volemia è rappresentato dalle dimensioni e dalle variazioni del diametro della vena cava inferiore (IVC). La dimensione massima della IVC non dovrebbe superare i $20 \mathrm{~mm}$. È stato dimostrato che le dimensioni della IVC sono l'espressione dello stato di volemia. Esiste, inoltre, una buona correlazione fra lo stato di volemia e i diametri della IVC a fine inspirazione e a fine espirazione, ottenuto, possibilmente, con M-mode. Questo parametro è noto come l'indice di collassabilità della IVC e viene calcolato come la differenza nel diametro del vaso in espirazione e in inspirazione diviso per il diametro espiratorio per 100. II valore ottenuto da questo calcolo deve superare il $50 \%$; un valore inferiore associato alla dilatazione del diametro della IVC è suggestivo per scompenso congestizio. I parametri per determinare lo stato di idratazione dovrebbero essere valutati durante il monitoraggio nel corso del trattamento sostitutivo e farmacologico del paziente con sovraccarico di volume, anche senza segni di scompenso congestizio; 
4. versamento pericardico: il sospetto di versamento pericardio (VP) è una comune indicazione clinica per la valutazione di un paziente uremico terminale o nel corso di un trattamento sostitutivo. La stima semiquantitativa eseguita in $M$-mode o in $B$-mode è, di solito, l'approccio adeguato per una corretta valutazione clinica del paziente. Un VP modesto è rilevabile a livello della parete posteriore o della parete libera del ventricolo destro. La raccolta globale di circa $1 \mathrm{~cm}$ intorno all'intera struttura cardiaca suggerisce un VP non inferiore ai 200 mL. II VP è, di solito, rilevabile nelle proiezioni sottocostale, parasternale e apicale, ma, in molti pazienti uremici con scarsa qualità della finestra acustica, la proiezione sottocostale rappresenta spesso I'unica alternativa valida. Gli effetti emodinamici del VP dipendono dalla relazione pressione-volume pericardica, dalla velocità in cui si forma la raccolta e dal volume del versamento;

5. valutazione morfologica e funzionale delle valvole cardiache: in situazioni di dispnea progressiva o di segni associati allo scompenso congestizio, la valutazione di grossolane alterazioni funzionali a carico del sistema valvolare può rendersi utile con il semplice impiego del box color-Doppler. Inoltre, utilizzando la modalità in B-mode, è possibile osservare il funzionamento complessivo delle cuspidi aortiche e il meccanismo di coaptazione dei lembi mitralici, controllando in real-time la presenza di vegetazioni o di filamenti utili per la diagnosi differenziale di endocardite batterica. La modalità $B$-mode consente anche di dare una stima qualitativa o semiquantitativa sulla presenza di calcificazioni o fibrocalcificazioni valvolari o dell'apparato sottovalvolare.

\section{Conclusione}

L'ecocardioscopia è una valutazione ecografica qualitativa mirata della morfologia e/o della funzione cardiaca eseguita da uno specialista cardiologo o non cardiologo.

L'utilizzo di questa metodica diagnostica sta diventando sempre più comune e ben consolidato in ambienti non cardiologici, come in quelli intensivisti, internisti e generalisti. Rappresenta un metodo preciso con il quale si ottiene un'informazione clinica utile in real-time, al letto del paziente e in modo non invasivo, utilizzando un'apparecchiatura di dimensioni contenute e di buon profilo tecnico. Attualmente, diverse società scientifiche incoraggiano lo specialista non cardiologo a percorrere un iter formativo per adeguare le competenze diagnostiche degli operatori e favorendo l'approccio clinico e terapeutico nei diversi settori clinici.

Gli scenari per l'applicazione clinica dell'ecocardioscopia in ambiente nefrologico sono numerosi e nessuno ha caratteristiche marginali. Per tale motivo, si stima che, nel prossimo futuro, il capitolo "ecocardioscopia" sarà inserito nel curriculum formativo di tutti i nefrologi ecografisti.

\section{Disclosures}

Financial support: No financial support was received for this submission.

Conflict of interest: The authors have no conflict of interest.

\section{Bibliografia}

1. Go AS, Chertow GM, Fan D, McCulloch CE, Hsu C. Chronic kidney disease and the risks of death, cardiovascular events, and hospitalization. N Engl J Med 2004;351(13):1296-305.

2. Collins AJ, Foley RN, Chavers B, et al. US Renal Data System 2013 Annual Data Report. Am J Kidney Dis 2014;63(1 Suppl.): A7.

3. Uhlig K, Levey AS, Sarnak MJ. Traditional cardiac risk factors in individuals with chronic kidney disease. Semin Dial 2003;16(2): 118-27.

4. Amann K, Rychlík I, Miltenberger-Milteny G, Ritz E. Left ventricular hypertrophy in renal failure. Kidney Int Suppl 1998;68:S78-85.

5. Bhan I, Thadhani R. Vascular calcification and ESRD: a hard target. Clin J Am Soc Nephrol 2009;4(Suppl. 1):S102-5.

6. Rennenberg RJ, Kessels AG, Schurgers LJ, van Engelshoven JM, de Leeuw PW, Kroon AA. Vascular calcifications as a marker of increased cardiovascular risk: a meta-analysis. Vasc Health Risk Manag 2009;5(1):185-97.

7. Manasia AR, Nagaraj HM, Kodali RB, et al. Feasibility and potential clinical utility of goal-directed transthoracic echocardiography performed by noncardiologist intensivists using a small hand-carried device (SonoHeart) in critically ill patients. J Cardiothorac Vasc Anesth 2005;19(2):155-9.

8. Beaulieu Y. Bedside echocardiography in the assessment of the critically ill. Crit Care Med 2007;35(5 Suppl.):S235-49.

9. Bierig SM, Ehler D, Knoll ML, Waggoner AD. American Society of Echocardiography minimum standards for the cardiac sonographer: a position paper. J Am Soc Echocardiogr 2006;19(5):471-4.

10. Spencer KT, Kimura BJ, Korcarz CE, Pellikka PA, Rahko PS, Siegel RJ. Focused cardiac ultrasound: recommendations from the American Society of Echocardiography. J Am Soc Echocardiogr 2013;26(6):567-81.

11. Neskovic AN, Edvardsen T, Galderisi M, et al. Focus cardiac ultrasound: the European Association of Cardiovascular Imaging viewpoint. Eur Heart J Cardiovasc Imaging 2014;15(9):956-60.

12. González-Juanateya JR, Diegob JJG de, Cosials JB. Echocardioscopy: Official Stance of the Spanish Society of Cardiology about operation of portable ultrasound equipments. CorSalud Rev Enfermedades Cardiovasc 2014;6(3):208-10.

13. SIEC - Società Italiana di Ecografia Cardiovascolare [Internet]. [cited 2016 May 3]. Available from: http://www.siec.it/.

14. Società Italiana di Nefrologia [Internet]. [cited 2016 May 3]. Available from: http://www.sin-italy.org/web/eventi/SIN/corsi.cfm? $p=$ ecografia_nefrologica\#.

15. Mueller X, Stauffer JC, Jaussi A, Goy JJ, Kappenberger L. Subjective visual echocardiographic estimate of left ventricular ejection fraction as an alternative to conventional echocardiographic methods: comparison with contrast angiography. Clin Cardiol 1991;14(11):898-902.

16. Floccari F, Granata A, Rivera R, et al. Echocardiography and right ventricular function in NKF stage III cronic kidney disease: Ultrasound nephrologists' role. J Ultrasound 2012;15(4): 252-6. 